\title{
Acquired von Willebrand syndrome in multiple myeloma
}

\author{
Irena Djunic ${ }^{1}$, Ivo Elezovic ${ }^{1}$, Vesna Ilic $^{2}$, Dragica Tomin ${ }^{1}$, Nada Suvajdzic- \\ Vukovic $^{1}$, Jelena Bila ${ }^{1}$, Darko Antic ${ }^{1}$, Ana Vidovic ${ }^{1}$, Nadezda Milosevic-Jovcic ${ }^{2}$
}

${ }^{1}$ Clinic for Haematology, Clinical Center of Serbia, Belgrade, Serbia, ${ }^{2}$ Institute for Medical Research, Belgrade, Serbia

Acquired von Willebrand syndrome (AvWS) is an uncommon complication of multiple myeloma (MM), and it is believed to be connected with paraprotein. The aim of this study was to determine the incidence of AvWS in patients with MM, and estimate the role of paraprotein in its occurrence. The study included 40 patients with MM. The plasma level of paraprotein, platelet adhesion on glass pearls, plasma von Willebrand factor antigen concentration, and ristocetin-induced platelet aggregation (RIPA) were measured initially. Absence of RIPA was found in six patients with MM (15\%); however, all six of them had normal levels of von Willebrand factor antigen. Paraprotein was isolated from the serum of these patients. Platelet aggregation was measured in six healthy donors before and after addition of the isolated paraprotein. RIPA was significantly decreased in healthy donors in the presence of paraprotein $(P<0.001)$. The same test was repeated with added human immunoglobulins for intravenous use without any change in RIPA. A significant negative correlation between plasma paraprotein level and RIPA was found $(P<0.001)$. These investigations have shown that paraprotein is associated with AvWS in patients with MM.

Keywords: Acquired von Willebrand syndrome, Multiple myeloma, Paraprotein

\section{Introduction}

Von Willebrand factor (vWF) is a high molecular weight glycoprotein (GP) that plays an important role in hemostasis by reacting with platelet membrane receptors. ${ }^{1}$ It is synthesized in endothelial cells and megakaryocytes. $^{2}$ At the site of vascular injury, binding of $\mathrm{vWF}$ to subendothelial collagen induces conformational changes in the vWF molecule that lead to platelet activation through GP Ib and GP IIb/ IIIa, the principal platelet vWF receptors. ${ }^{2-4}$

Qualitative or quantitative abnormalities of $\mathrm{vWF}$ result in von Willebrand disease (vWD), the most common inherited bleeding disorder transmitted as an autosomal, most frequently dominant trait, with prevalence of $1-2 \%$ in the general population. ${ }^{5}$ There are three main subtypes of vWD: (i) type $1 \mathrm{vWD}$ is partial deficiency of qualitatively normal vWF; (ii) type 2 vWD is caused by functionally abnormal vWF; and (iii) type 3 vWD represents a virtual absence of vWF protein. ${ }^{2,5,6}$ Concerning laboratory evaluation of vWD, due to assay limitations and vWD heterogeneity, no single laboratory procedure can detect all forms of vWD. However, various panels are used in different laboratories, depending

Correspondence to: Irena Djunic, Clinic for Haematology, Clinical Center of Serbia, Koste Todorovica 2, 11000 Belgrade, Serbia. Email: irenadju@ eunet.rs on preference and availability. The classical test panels for laboratory evaluation of vWD are: plasma level of vWF antigen (vWF:Ag), vWF:ristocetin cofactor activity, factor VIII:coagulant activity, vWF:collagenbinding activity, ristocetin-induced platelet aggregation (RIPA), vWF:multimers, and vWF:factor VIII binding assay. $^{7}$

In contrast to congenital disease, acquired von Willebrand syndrome (AvWS) is a rare disorder, most commonly associated with various underlying diseases, with an estimated prevalence ranging from 0.04 to $0.13 \%$ in the general population. ${ }^{3,8}$ The pathophysiology of AvWS is not completely understood. ${ }^{8}$ Several pathogenic mechanisms have been proposed: (i) specific inhibitory antibodies to vWF; (ii) non-neutralizing autoantibodies to vWF (immune complex); (iii) adsorption of vWF by malignant cells; (iv) increased $\mathrm{vWF}$ proteolytic degradation; and (v) decreased vWF synthesis. ${ }^{3,8-15}$

Most patients with AvWS (47\%) had an underlying lymphoproliferative or plasma cell proliferative disorder. ${ }^{3}$ Multiple myeloma (MM) is a malignant plasma cell neoplasia characterized by the proliferation of a single clone of plasma cells derived from $\mathrm{B}$ cells in the bone marrow. ${ }^{16}$ The plasma cell clone produces a monoclonal $(\mathrm{M})$ protein, known as the M-component, or paraprotein. ${ }^{16}$ It is believed that 
AvWS in MM is connected with paraprotein. ${ }^{3,17}$ Proposed possible pathophysiologic mechanisms, in rare published investigations, include an inhibiting role of paraprotein by binding to $\mathrm{vWF}$, or platelets $\mathrm{vWF}$ receptors, and adsorption of $\mathrm{vWF}$ by myeloma cells because they show aberrant expression of GP Ib. $3,8,12$

The aim of this study was to estimate the incidence of AvWS in patients with MM and the role of paraprotein in its origin.

\section{Materials and Methods}

This research included 40 patients with MM, among whom 29 were newly diagnosed and were in relapse. The characteristics of the patients are shown in Table 1. All patients were evaluated before treatment, and they received no antiaggregant or anticoagulant therapy. Only patients with a normal platelet count $\left(>150 \times 10^{9} / 1\right)$ were included. The following tests were initially performed in all of them: bleeding time (BT), prothrombin time (PT), partial thromboplastin time (PTT), platelet adhesion, plasma level of vWF:Ag, and RIPA. The concentration and type of paraprotein were determined according to the diagnostic criteria for MM from Durie et al. ${ }^{18}$ Paraprotein was separated from six patients who had complete absence of RIPA. Isolated paraprotein was added in plasma from six healthy donors and the RIPA test was repeated. In order to exclude the possibility of non-specific binding of paraproteins to platelet receptors in vitro, the platelet aggregation test was repeated again after adding immunoglobulins for intravenous (i.v.) use to plasma from the same healthy donors.

Routine hemostatic tests, PT and PTT, were determined on the coagulometer (Automated Coagulation Laboratory). The control values for PT were $75-120 \%$ (expressed as a percentage of the value for normal plasma). For PTT, the values were $25-35$ seconds. BT was measured according to the Duke method and the normal values were $60-180$ seconds.

Plasma level of vWF:Ag was determined with an enzyme-linked immunosorbent assay and the normal range was $50-150 \%$.

Platelet adhesion was measured by platelet retention on glass pearls by Salzman's method. ${ }^{19}$ Blood

Table 1 Patients characteristics

\begin{tabular}{lcc}
\hline Patient characteristics & No. of patients & $\%$ \\
\hline Total & 40 & \\
Diagnosis & 29 & 72.5 \\
$\quad$ Newly diagnosed & 11 & 27.5 \\
$\quad$ Relapsed & 18 & \\
Sex & 22 & 45 \\
$\quad$ Male & & 55 \\
$\quad$ Female & 60.1 & \\
Age (years) & \\
$\quad$ Median & $39-82$ & \\
$\quad$ Range & \\
\hline
\end{tabular}

was taken directly from a vein $(N)$ into a test tube, while from another vein, blood was taken through a system filled with glass pearls $(n)$. Platelet adhesion was determined according to the following formula: $[(N-n) / N] \times 100$ and expressed as a percentage of adhered platelets. Normal values are $20-60 \%$.

Platelet aggregation was measured by Born's optical method. ${ }^{20}$ A sample of venous blood was taken into a test tube containing anticoagulant sodium citrate in the ratio 1:9. Plasma rich in platelets was obtained by centrifugation at 800 rotations/minute for 15 minutes. RIPA was determined by adding $50 \mu \mathrm{l}$ of ristocetin in concentration $1.2 \mathrm{mg} /$ $\mathrm{ml}$ in $450 \mu \mathrm{l}$ of plasma rich in platelets. Aggregation was recorded as the increase in light transmission through the mixture. The maximum aggregation achieved, expressed as transmission unit percentage, was determined from the aggregation curve graphic record. Normal values of aggregation are $60-100 \%$ (0.6-1.0).

The concentration and type of paraproteins were determined by horizontal electrophoresis in 1\% agar gel at the electric field strength of $8 \mathrm{~mA}$.

Paraproteins were partially fractionated from serum by adding Rivanol solution (1.5\%) to the serum in the volume ratio $1: 1$. The mixture was left overnight at $4{ }^{\circ} \mathrm{C}$, and then centrifuged for 10 minutes at 3000 rotations/minute. The supernatant contains IgG (so-called Rivanol-soluble IgG). The protein concentration in the samples of isolated $\operatorname{IgG}$ was determined by the tannin turbidimetric method. The relative abundance of protein fractions was obtained by densitometry of electrophoretically separated fractions, using ImageQuant software (Molecular Dynamics, San Diego, CA, USA). Horizontal electrophoresis of $\operatorname{IgG}$ was performed in $1 \%$ agar gel at the field strength of $8 \mathrm{~mA}$ per sample for 30 40 minutes. Protein fractions were visualized by staining with amido-black. The subclass of isolated IgG paraproteins was determined by western blot method. After electrophoretic separation of $\mathrm{IgG}$ on agar gel, the proteins were transferred to a nitrocellulose membrane and allowed to dry in the air. All membranes were incubated with mouse monoclonal antibodies specific for gamma 1 , gamma, gamma $_{3}$, and gamma 4 heavy chains of human $\mathrm{IgG}$. Reaction was visualized with a secondary antibody (goat antibody to mouse $\mathrm{IgG}$ labeled with peroxidase) and chloronaphthol as the substrate for peroxidase, which, in the presence of $\mathrm{H}_{2} \mathrm{O}_{2}$, gives a blue-purple precipitate. $^{21-23}$

The interferential statistical procedures used here are methods for estimating the significance of differences: Student's $t$-test for independent samples, chi-square test, Fisher's exact significance test, and correlation methods (Pearson's coefficient of linear 


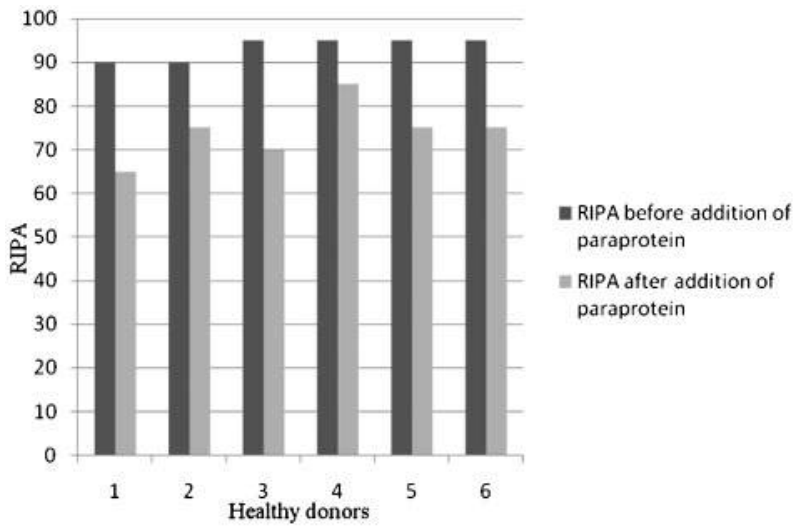

Figure 1 RIPA was significantly decreased after adding paraproteins to plasma from healthy donors $(P<0.001)$.

correlation and Spearman's coefficient of range correlation). Statistical significance was set at $P \leqslant 0.05$.

\section{Results}

Among our 40 patients with MM, RIPA was absent in six of them (15\%); more exactly, their level of RIPA was $0 \%$. Four patients were female and two were male. The plasma level of vWF:Ag was in the normal range in all of them. Decreased platelet adhesion was found in two patients with absent RIPA, disturbed PT in one of them, while BT and PTT were in the normal range in all of them. Two patients with AvWS had an evident bleeding syndrome, one with massive skin hematomas and the other with prolonged epistaxis. Laboratory findings for the six patients with AvWS are summarized in Table 2.

Paraprotein of the IgG class was detected in 34 $(85 \%)$ and $\operatorname{IgA}$ in six $(15 \%)$ patients enrolled in this study. All six patients with AvWS had IgG class paraprotein, three with the lambda subclass and three with the kappa subclass. Serum paraprotein concentrations were negatively correlated with RIPA $(P<0.001$, rho $=-0.671)$.

RIPA was measured in six healthy donors before and after addition of paraprotein isolated from the serum of patients with absent RIPA, in vitro. The change in the average value of the maximal level of RIPA before and after adding paraproteins to plasma from healthy donors was statistically significant ( $t=8.032, \mathrm{df}=5, P<0.001 ;$ Fig. 1$)$. In contrast to

Table 2 Laboratory findings of six patients with AvWS

\begin{tabular}{lllllrl}
\hline $\begin{array}{l}\text { Case } \\
\text { no. }\end{array}$ & $\begin{array}{l}\text { RIPA } \\
\text { (\%) }\end{array}$ & $\begin{array}{l}\text { vWF:Ag } \\
\text { (\%) }\end{array}$ & $\begin{array}{l}\text { Platelet } \\
\text { adhesion (\%) }\end{array}$ & $\begin{array}{l}\text { BT } \\
\text { (seconds) }\end{array}$ & $\begin{array}{l}\text { PT } \\
(\%)\end{array}$ & $\begin{array}{l}\text { PTT } \\
(\%)\end{array}$ \\
\hline 1 & 0 & 84 & 37 & 105 & 81 & 31.8 \\
2 & 0 & 112 & 36 & 75 & 71 & 29.9 \\
3 & 0 & 70 & 60 & 150 & 53 & 32.3 \\
4 & 0 & 84 & 40 & 75 & 96 & 25 \\
5 & 0 & 70 & 7.5 & 105 & 106 & 31.6 \\
6 & 0 & 84 & 5 & 165 & 70 & 35 \\
\hline
\end{tabular}

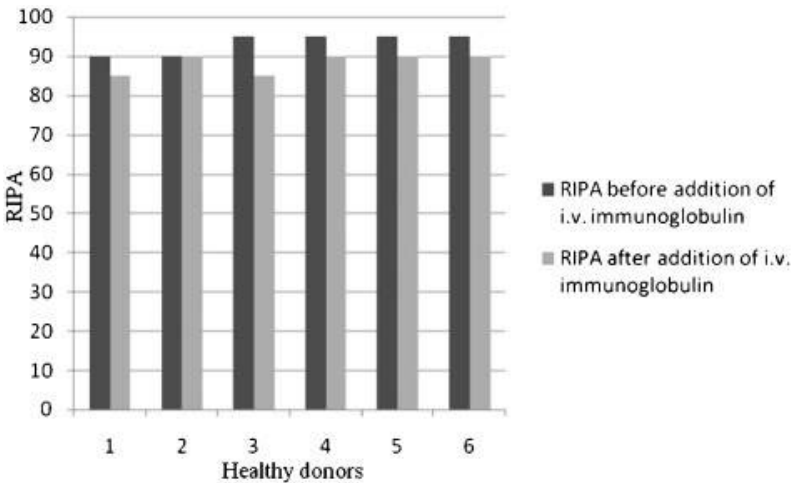

Figure 2 RIPA was not significantly changed after adding immunoglobulins for intravenous use to plasma of healthy donors.

these results, after the addition of immunoglobulins for i.v. use to plasma of the same healthy donors, there was no significant change in RIPA (Fig. 2).

Three patients achieved complete remission of $\mathrm{MM}$, i.e. after disappearance of paraprotein from their serum, repeated RIPA tests were normalized.

\section{Discussion}

AvWS is reported in association with monoclonal gammaglobulins, lymphoproliferative disorders such as lymphomas, myelomas, and Wilms' tumors, and autoimmune disorders, suggesting deficiency of an immunological etiology. ${ }^{4}$ Laboratory findings concerning AvWS coexisting with these diseases are similar to those for inherited type $2 \mathrm{~A}$ vWD. ${ }^{17}$ The monoclonal protein, paraprotein, in some patients appeared to have specificity against vWF. ${ }^{3}$ Paraprotein inhibited the function of $\mathrm{vWF}$ by binding to the vWF Al domain, which is the GP Ib binding domain. ${ }^{17}$ Moreover, Shiganawa et al. showed that paraprotein inhibited RIPA by interference with the A1 domain of vWF and directly bound the 39/34-kD fragment of vWF that encompasses this domain. ${ }^{24}$ Impaired platelet function due to abnormal platelet aggregation has been associated with non-specific coating of platelet receptors by paraprotein. ${ }^{17}$ Paraprotein can also inhibit platelet function by specific binding to the $\mathrm{vWF}$ platelet receptor GP Ib, thereby inhibiting binding of $\mathrm{vWF}$ to ristocetinstimulated platelets, and by binding to platelet IIb/ IIIa receptors. ${ }^{17,25,26}$

Among our 40 patients with MM, six (15\%) exhibited absence of RIPA but had normal plasma levels of vWF:Ag and normal BT and PTT. Most of them also had normal PT (five of six), but two subjects showed decreased platelet adhesion. In accordance with these data, our patients also had type 2 AvWS, i.e. a qualitative dysfunction of vWF. A normal plasma level of $\mathrm{vWF}: \mathrm{Ag}$ is specific for type 2 vWD, the most common type of AvWS in plasma cell dyscrasias. ${ }^{7,17}$ BT, PT, and PTT are not specific tests 
for vWD, and they are probably better used as a more global screening assay for potential hypocoagulopathies that might include vWD. ${ }^{7}$ Inhibitors of vWF have been identified most frequently by inhibition of RIPA. ${ }^{27}$ The antibiotic ristocetin facilitates GPIb-mediated platelet binding of vWF and provides a functional assessment of vWF. In two patients with AvWS, platelet adhesion was decreased, which showed that vWF is dysfunctional.

The actual incidence of AvWS in MM has not been precisely estimated. In the study of Mohri et al., ${ }^{4}$ AvWS was diagnosed in three out of 42 patients with $\mathrm{MM}$, while an inhibitor to $\mathrm{vWF}$ was identified in eight patients out of 25 with AvWS. ${ }^{4}$

In our investigation, paraprotein inhibited $\mathrm{vWF}$ in six patients. All paraprotein were IgG class, three IgGlambda subclass and three IgGkappa subclass. Most inhibitors described earlier belonged to the IgGkappa subclass of paraproteins. ${ }^{8}$ We showed that paraprotein inhibited $\mathrm{vWF}$ leading to the type $2 \mathrm{~A}$ AvWS in patients with MM. Thus, paraprotein from serum of these six patients with absence of RIPA significantly decreased RIPA in plasma from six healthy donors, in vitro. Addition of immunoglobulins for i.v. use to healthy plasma nullified the effect and so the possibility that paraprotein bound nonspecifically to platelet receptors was excluded. These findings were supported by repeating RIPA in three patients who had achieved complete remission of MM, i.e. when paraprotein was not detectable in their serum after a treatment, RIPA was normalized in all three patients. Another finding in our study supporting the importance of paraprotein in the origin of AvWS was the significant negative correlation between serum paraprotein level and RIPA.

\section{Conclusion}

This investigation showed that in patients with MM, paraprotein caused AvWS, type 2A, either by binding to vWF or by specific binding to vWF platelet receptors.

\section{References}

1 Ruggeri ZM, Zimmerman RS. Von Willebrand factor and von Willebrand disease. Blood 1987;70:895-904.

2 James PD, Notley C, Hegadorn C, Leggo J, Tuttle A, Tinlin S, et al. The mutual spectrum of type 1 von Willebrand disease: results from a Canadian cohort study. Blood 2007;109:145-54.

3 Kumar S, Pruthi RK, Nichols WL. Acquired von Willebrand disease. Mavo Clin Proc 2002;77:181-7.

4 Mohri H, Motomura S, Kanamori H, Matsuzaki M, Watanabe $\mathrm{S}$, Maruta A, et al. Clinical significance of inhibitors in acquired von Willebrand syndrome. Blood 1998;91:3623-9.

5 Sadler JE. A revised classification of von Willebrand disease. Thromb Haemost 1994;71:520-5.

6 Sadler JE, Mannucci PM, Berntorp E, Bochkov N, Boulyjenkov V, Ginsburg D, et al. Impact, diagnosis and treatment of von Willebrand disease. Thromb Haemost 2000;84:160-74.
7 Favaloro EJ. Laboratory identification of von Willebrand disease: technical and scientific perspectives. Semin Thromb Hemost 2006;32:456-71.

8 Mohri H. Acquired von Willebrand syndrome: features and management. Am J Hematol 2006;81:616-23.

9 Mohri H, Hisanaga S, Mishima A, Fujimoto S, Uezono S, Okubo T. Autoantibody inhibits binding of von Willebrand factor to glycoprotein $\mathrm{Ib}$ and collagen in multiple myeloma: recognition sites present on the $\mathrm{A} 1$ loop and $\mathrm{A} 3$ domains of von Willebrand factor. Blood Coagul Fibrinolvsis 1998;9:91-7.

10 van Genderen PJ, Vink T, Mishiels JJ, van 't Veer MB, Sixma $\mathrm{JJ}$, van Vliet HH. Acquired von Willebrand disease caused by an autoantibody selectively inhibiting the binding of von Willebrand factor to collagen. Blood 1994;84:3378-84.

11 Scrobohaci ML, Daniel MT, Levy Y, Marolleau JP, Brouet JC. Expression of GpIb on plasma cells in a patient with monoclonal $\operatorname{IgG}$ and acquired von Willebrand disease. $\mathrm{Br} \mathrm{J}$ Haematol 1993;84:471-5.

12 Richard C, Cuadrado MA, Prieto M, Batlle J, López Fernández MF, Rodriguez Salazar ML, et al. Acquired von Willebrand disease in multiple myeloma secondary to absorption of von Willebrand factor by plasma cells. Am J Hematol 1990;35:114-7.

13 Tefferi A, Hanson CA, Kurtin PJ, Katzmann JA, Dalton RJ, Nichols WL. Acquired von Willebrand's disease due to aberrant expression of platelet glycoprotein $\mathrm{Ib}$ by marginal zone lymphoma cells. Br J Haematol 1997;96:850-3.

14 Budde U, van Genderen PJ. Acquired von Willebrand disease in patients with high platelet counts. Semin Thromb Hemost 1997;23:425-31.

15 Pareti FI, Lattuada A, Bressi C, Zanobini M, Sala A, Steffan A, et al. Proteolysis of von Willebrand factor and shear stressinduced platelet aggregation in patients with aortic valve stenosis. Circulation 2000;102:1290-5.

16 Kyle RA, Rajkumar SV. Criteria for diagnosis, staging, risk stratification and response assessment of multiple myeloma. Leukemia 2009;23:3-9.

17 Eby CS. Bleeding and thrombosis risks in plasma cell dyscrasias. Hematol Am Soc Hematol Educ Program 2007: $158-64$.

18 Durie BG, Kyle RA, Belch A, Bensinger W, Blade J, Boccadoro $\mathrm{M}$, et al. Myeloma management guidelines: a consensus report from the Scientific Advisors of the International Myeloma Foundation. Haematol J 2003;4:379-98.

19 Salzman EW. Measurement of platelet adhesiveness. A simple in vitro technique demonstrating an abnormality in von Willebran's disease. J Lab Clin Med 1963;62:724-35.

20 Born GV, Gross MJ. The aggregation of blood platelets. J Physiol 1963;168:178-95.

21 Sagan Z. The application of Rivanol for serum transferin and immunoglobulin G determination. Clin Chem Acta 1968; $21: 225-31$

22 Johansen BG. Agarose gel electrophoresis. Scand J Clin Lab Invest 1972;124:7-9.

23 Mejbaum-Katzenellenbogen W. Turbidimetric micromethod of determination of proteins with tannin. Acta Biochim Pol 1955;2:279-94.

24 Shinagawa A, Kojima H, Bernt MC, Kaneko S, Suzukawa K, Hasegawa $\mathrm{Y}$, et al. Characterization of a myeloma patient with a life-threatening hemorrhagic diathesis: presence of a lambda dimer protein inhibiting shear-induced platelet aggregation by binding to the A1 domain of von Willebrand factor. Thromb Haemost 2005;93:889-96.

25 Mohri H, Tanabe J, Ohtsuka M, Yoshida M, Motomura S, Nishida S, et al. Acquired von Willebrand disease associated with multiple myeloma: characterization of an inhibitor to von Willebrand factor. Blood Coagul Fibrinolysis 1995;6:561-6.

26 Veyradier A, Jenkins CS, Fressinaud E, Meyer D. Acquired von Willebrand syndrome: from pathophisiology to management. Thromb Haemost 2000;84:175-82.

27 Fricke WA, Brinkhous KM, Garris JB, Roberts HR. Comparison of inhibitory and binding characteristics of an antibody causing acquired von Willebrand syndrome: an assay for von Willebrand factor binding by antibody. Blood 1985;66:562-9. 